\title{
Transoral laser microsurgery for oral squamous cell carcinoma: Oncologic outcomes and prognostic factors
}

\author{
Parul Sinha, MBBS, MS ${ }^{1}$, Trevor Hackman, MD², Brian Nussenbaum, MD¹, Ningying Wu, \\ $\mathrm{PhD}^{3}$, James S. Lewis Jr, MD ${ }^{1,4}$, and Bruce H. Haughey, MBChB, MS, FRACS ${ }^{1, *}$ \\ ${ }^{1}$ Department of Otolaryngology - Head and Neck Surgery, Washington University School of \\ Medicine, St. Louis, Missouri \\ ${ }^{2}$ Department of Otolaryngology - Head and Neck Surgery, University of North Carolina, Chapel \\ Hill, North Carolina \\ ${ }^{3}$ Department of Biostatistics, Washington University School of Medicine, St. Louis, Missouri \\ ${ }^{4}$ Department of Pathology and Immunology, Washington University School of Medicine, St. Louis, \\ Missouri
}

\begin{abstract}
Background-Modest survival rates are published for treatment of oral squamous cell carcinoma (OSCC) using conventional approaches. Few cohort studies are available for transoral resection of OSCC.
\end{abstract}

Methods-Analysis for recurrence, survival, and prognosis of patients with OSCC treated with transoral laser microsurgery (TLM) \pm neck dissection was obtained from a prospective database.

Results-Ninety-five patients (71 patients had stages T1-T2 and 24 had stages T3-T4 disease) with minimum follow-up of 24 months met criteria and demonstrated negative margins in $95 \%$. Five-year local control (LC) and disease-specific survival (DSS) were 78\% and 76\%, respectively. Surgical salvage achieved an absolute final locoregional control of $92 \%$. Immune compromise and final margins were prognostic for LC, whereas T classification, N classification, TNM stage, comorbidity, and perineural invasion were also significant for DSS.

Conclusion-We document a large series of patients with OSCC treated with TLM, incorporating T1 to T4 primaries. A significant proportion of stage III/IV cases demonstrates feasibility of TLM in higher stages, with final margin positivity of 5\%, LC greater than $90 \%$, and comparable survival outcomes.

\section{Keywords}

oral cancer; tongue; transoral microsurgery; laser; survival

\footnotetext{
(C) 2013 Wiley Periodicals, Inc.

*Corresponding author: B. H. Haughey, Department of Otolaryngology Head and Neck Surgery, 660 S. Euclid Avenue, Campus Box 8115, St. Louis, MO 63110. haugheyb@ent.wustl.edu.

This work was presented at the 8th International Conference on Head and Neck Cancer. Toronto, Canada, July 24, 2012.

The authors disclosed no conflicts of interest.
} 


\section{INTRODUCTION}

Surgery performed with curative intent is a widely established treatment modality for oral cancer, with the addition of adjuvant therapy in advanced cases. ${ }^{1}$ However, within the gamut of head and neck cancer, surgical resection of oral squamous cell carcinoma (OSCC) poses unique therapeutic challenges not only because of the aggressive behavior of these tumors, but also the potential for functional and esthetic deficits. These considerations promote a transition from the long-standing practice of transfacial or transcervical external composite resection to minimally invasive resections without compromising oncologic outcomes. In the presence of certain experimental ${ }^{2}$ and clinical ${ }^{3,4}$ evidence that incontinuity neck dissection(s) were not inferior to discontinuity approaches, interest in transoral resections was rekindled in the late 1970s. Coupling of the carbon dioxide laser to the operating microscope was the harbinger of a surgical technique - transoral laser microsurgery (TLM). ${ }^{5}$ The first series of 57 oral cancer transoral laser resections was reported by Strong and $\mathrm{Jako}^{4}$ followed by a further United States report on outcomes for transoral laser resection for OSCC by Panje et al. ${ }^{6}$

With technology improvements, TLM has found wide application in head and neck disease and has become a preferred organ-preservation surgical approach for laryngeal ${ }^{7}$ and oropharyngeal cancer. ${ }^{8}$ It is particularly suited for OSCC in which the primary tumor is resected to a carefully monitored negative histologic margin while disease-free, surrounding tissue and important structures are maximally preserved. Higher volumes of preserved normal tissue correlate with better articulation, mastication, and swallowing function, particularly for primaries of the oral tongue.

Few reports are available for OSCC treated exclusively with TLM. Available literature mainly delineates the use of TLM techniques for early stage tumors, the data remaining is particularly sparse for high $\mathrm{T}$ classification lesions. The largest series to date is limited to a report of $\mathrm{T} 1$ and $\mathrm{T} 2 \mathrm{~N} 0$ stage patients $(\mathrm{n}=90)$ with OSCC, with an observed recurrence rate of $12 \% .{ }^{9}$ TLM for higher T classifications is documented in a smaller cohort $(n=64)$, all but with staged neck dissection(s). ${ }^{10}$ The latter study reported a recurrence rate of $36 \%$, with 5 -year tumor-related survival estimates of $81 \%$ for stages I to II, $73 \%$ for stage III, and $21 \%$ for stage IV tumors. Non-TLM surgical studies for all-stage OSCC report 5-year diseaserelated survival estimates from $42 \%$ to $76 \%$, and recurrence rates of $15 \%$ to $30 \%$. $^{11-15}$

Factors prognostic for OSCC treated with non-TLM approaches have not been validated or refuted in a TLM series. Because TLM is performed with the goal of rendering patients completely free of known gross and microscopic disease at the primary site, it is important to identify variables that can reliably predict recurrences and determine the need for adjuvant therapy in TLM-treated cases.

In our study, we present a large OSCC cohort in which TLM technique has been applied to each subsite and stage of oral primary, inclusive of cases with nodal metastasis. Our objective was to evaluate the effectiveness of the TLM approach for management of OSCC by documentation of oncologic and survival outcomes. We also describe the variables that are prognostic for these outcomes and for locoregional control of disease.

\section{MATERIALS AND METHODS}

\section{Study design, population, and patient selection}

A prospectively assembled, computerized, database (Excel; Microsoft, Redmond, WA) comprising patients with head and neck cancer treated with TLM from May 1995 through July 2010 at Washington University Medical School of Medicine in St. Louis, Missouri, was 
searched for patients with OSCC: all had undergone TLM with curative intent for histologically confirmed oral malignancy. The Human Research Protection Office at Washington University Medical School of Medicine approved data collection for the transorally treated patients and individual consent was obtained from patients for entry of their information into the database.

\section{Inclusion and exclusion criteria}

The fundamental inclusion criteria for this study were: (1) previously untreated, histologically proven OSCC; (2) primary treatment with TLM \pm neck dissection \pm adjuvant therapy; (3) minimum follow-up of 24 months or to death; and (4) second primary OSCC, if the index tumor was not in the oral cavity, with no radiation administered anywhere in the head and neck region.

Exclusion criteria were: (1) recurrent tumor failing previous surgery, radiotherapy, or chemotherapy; (2) distant metastasis detected at presentation; (3) patients alive but with a follow-up of less than 24 months; (4) second primary OSCC in which the previous index primary was in the oral cavity, and/or the treatment modality included radiotherapy to the head and neck region.

Our study's population composes $54.8 \%$ of patients with OSCC who underwent surgery during the study period. The primary contraindications for the TLM approach were advanced tumors with sufficient mandibular involvement to necessitate a segmental mandibulectomy, facial skin invasion, retromolar trigone tumors with significant extension to the infratemporal fossa, floor of mouth (FOM) tumors with extension outside the mylohyoid, and upper alveolar ridge tumors with extension into the maxillary sinus that would require more than transoral infrastructure maxillectomy.

\section{Data collection}

Demographic data, clinical variables including disease staging, treatments, and complications, pathologic variables, and follow-up data on disease and functional outcomes, for each patient were recorded in the TLM database as they transpired. All information from these prospectively collected data was verified by a careful scrutiny of medical records. The follow-up data were further confirmed through searches in national death registries and direct telephone contact with patients/family members. Comorbidities at the time of first office presentation with a diagnosis of oral cancer were retrospectively captured using the validated instrument, Adult Comorbidity Evaluation-27 (ACE-27). ${ }^{16}$ Also recorded was presence of or history of immune compromise at the time of OSCC diagnosis. Patients were considered to be immune compromised for the purpose of this analysis if they had a history of intrinsic or iatrogenic immunosuppression, hematopoietic disorders (eg, lymphoma), autoimmune disorders, immunoglobulin deficiency, history of chemoradiation for 2 or more solid organ cancers, AIDS, and chronic debilitating illnesses, such as uncontrolled diabetes or cirrhosis. Smoking status (never vs ever) and life-time tobacco exposure in number of pack-years (light vs heavy smoking) was determined using information from the clinician's history, physical notes, and the patient's self-reported health history completed at the time of presentation. Patients were classified as heavy smokers if the lifetime tobacco exposure was $\geq 20$ pack-years. ${ }^{17,18}$

Treatment-related complications and days of hospitalization were recorded, as were functional outcomes (tracheostomy rates and swallowing function, as estimated by the Functional Outcome Swallowing Scale (FOSS) ${ }^{19}$ at the follow-up). 


\section{Tumor and nodal staging}

The 7th edition American Joint Committee on Cancer's staging manual was used. T classification was determined primarily by clinical staging, unless operative findings or pathology results upstaged the disease because of more accurate measurement of tumor size or reporting of bone invasion. $\mathrm{N}$ classification was determined clinically and/or from pathology reports for patients who underwent neck dissections.

\section{Treatments}

Preoperative assessment-Especially in oral tongue and FOM lesions, office ultrasonography for primary tumor depth and neck nodal status was frequently performed by the operating surgeon. For early tongue cancers (T1-T2), our criterion for elective neck dissection was $4 \mathrm{~mm}$ of tumor thickness ${ }^{20}$; advanced primaries (T3-T4) required elective neck dissection irrespective of tumor thickness. CT or MRI was also requested for assessment of the primary, mainly for large endophytic/infiltrative-appearing lesions or in patients with severe trismus, and to evaluate cervical involvement in patients with a thick neck. Distant metastatic workup using CT, positron emission tomography, or positron emission tomography-CT was performed according to probability thereof.

Transoral laser microsurgery for primary tumor-Resection of the primary tumor was performed using the principles of TLM, described earlier by Steiner et al. ${ }^{21}$ In brief, optimum visualization of the primary tumor is achieved using lip retractors plus ratcheted or side-biting mouth props, and side-positioning of an operating microscope. The carbon dioxide laser is delivered via a hollow, mirror-lined handheld device; when necessary, large bulky tumors are cored out with cautery or laser to leave a thin "bowl" of tumor at the perimeter, followed by radial transtumoral cuts at several intervals well into normal tissue to assess deep tumor extent at multiple locations. TLM resection is carried out until all primary site disease is eliminated extending to a normal appearing tissue margin of approximately 1 $\mathrm{cm}$, when appropriate. The margin was $<1 \mathrm{~cm}$ margin around the tumor edge when an adjacent vital structure (eg, the main trunk of the lingual nerve or the proximal lingual artery) was closer than $1 \mathrm{~cm}$ to the tumor, but a peripheral zone of normal tissue was observed under the microscope, confirmed by intraoperative frozen section. For tumors posterolateral in location or extension, if radial incisions through the tumor demonstrated proximity to the submandibular gland, the superior portion of the gland was included with the primary resection. Reconstruction of a small through-and-through defect in the FOM was accomplished after the neck dissection with adjacent tissue transfer and digastric muscle flap transposition, reinforced by a cadaveric acellular graft, if required.

For FOM or alveolar ridge tumors that abut the bone, transverse transtumoral laser cuts were made that extended from the soft tissue side of the tumor, terminating submucoperiosteally at or even the bone. The purpose of these cuts was to assess the depth and radial extent of the tumor. Soft tissue components of the tumor were then removed and margins confirmed by frozen section. The alveolar bone was thus exposed, and then evaluated. Transoral ostectomy cuts were then made to excise bone adjacent to, or focally invaded by the tumor, typically a marginal or lingual plate mandibulectomy. The marrow contents from the margins were curetted and sent for frozen-section evaluation.

The surgical defect at the primary site was allowed to heal either by secondary intention or reconstructed using mucosal advancement flaps, split skin grafts, acellular cadaveric dermal grafts, or microvascular free flaps, depending on defect size and extent of any neck communication. 
Neck dissection-In patients with clinically positive necks or a high risk of occult metastasis, neck dissections were performed in the same surgical session with the TLM. Levels I through IV lymph nodes were removed in all dissections, with extended dissections when necessary. Tumors of the oral tongue or FOM approaching or extending across the midline necessitated contralateral elective neck dissection in the same or a staged session.

Adjuvant therapy-The decision for administration of adjuvant therapy was made in a multidisciplinary tumor board, based on pathology reports of extracapsular spread (ECS), 2 or more positive or contralateral lymph nodes, positive margins, patient choice, surgeon recommendation, and other laboratory and performance status criteria. Postoperative radiotherapy, when used in the presence of "high-risk" criteria such as positive surgical margins, presence of extracapsular spread (ECS), >1 metastatic node, perineural and/or lymphovascular invasion, was usually administered at a dose of 66 Gy with 220 cGy per fraction for 30 fractions over a 6-week period. In the absence of "high-risk" factors, the dose was reduced to $60 \mathrm{~Gy}$. For chemoradiotherapy, cisplatin was administered at $100 \mathrm{mg} / \mathrm{m}^{3}$ on days 1, 22, and 43 of the radiotherapy. Eight weekly doses of cetuximab were given in patients who were intolerant of cisplatin-related toxicity.

\section{Pathology}

All cases were reviewed to confirm the diagnosis of squamous carcinoma or variants $(n=5)$ thereof. A wide variety of variables with potential for prognostic impact were reviewed. Surgical margin status, primary tumor depth (in millimeters), advancing front of the tumor (pushing/infiltrating), tumor grade (well/moderately/poorly differentiated), and inflammatory response (absent/sparse/brisk) were recorded from the pathologist's reports. Also recorded was the presence or absence of perineural invasion, lymphovascular invasion, nodal involvement, ECS, and soft tissue metastasis along with the number of metastatic nodes.

\section{Follow-up}

Patients were followed up per clinical routine. All patients were followed 4 to 6 weeks after the initial postoperative care was completed. The intervals for follow-up visits were modified as deemed necessary for optimum disease surveillance but were usually spaced at 3 to 4 monthly intervals until the end of the first year; every 4 to 6 months in the second year, every 6 to 9 months in the third and fourth years, and annually thereafter. Patients were carefully monitored for any possible evidence of locoregional recurrence and distant metastasis. The evaluation consisted of (1) complete clinical examination of the head and neck, (2) annual chest imaging, usually CT, (3) head and neck imaging for cases difficult to assess clinically or suspected of having locoregional recurrence, and (4) biopsy or cytology of suspect recurrent lesions. All cases of recurrence were confirmed by biopsy. At each of the follow-up visits, the functional status regarding speech and swallowing rehabilitation was assessed and recorded.

\section{Study endpoints}

The primary endpoints for this study were local control (LC), disease-specific survival (DSS), and patterns of failure (local, regional, or distant). The secondary endpoints included recurrence-free survival (RFS) and overall survival (OS). Local recurrence was defined as biopsy proven tumor in the immediate vicinity (ie, within $2 \mathrm{~cm}$ ) of the original primary. Regional recurrence was defined as development of histologically proven metastasis in cervical lymph nodes. After completion of treatment, image or biopsy detected recurrent disease at sites away from the head and neck were defined as distant metastasis. 
LC was defined as the time between surgery and development of local recurrence. DSS was measured from the time of surgery to the date of death from oral cancer or the direct effects of its treatment. RFS was defined as the time from surgery to development of first disease recurrence - local, regional, distant, or death as a result of the disease. OS was estimated from the time from surgery to the date of death because of any cause.

\section{Statistical analysis}

A variety of factors known to have prognostic significance in head and neck squamous cell carcinoma, including patient demographics, site and stage of tumor, pathologic variables such as surgical margins, ECS, and adjuvant treatment modality were tested for significance of effects on the survival outcomes evaluated in this study. The data were analyzed using SAS 9.2 software (SAS Institute, Cary, NC). Heterogeneity between any 2 groups was investigated using chi-square or Fischer exact test for categorical data and independent $t$ test or its nonparametric equivalent Mann-Whitney $U$ test for continuous data. All statistical tests used were 2 -sided. Survival probability was estimated by Kaplan-Meier analysis and survival curves were compared by use of log-rank statistic. Five-year Kaplan-Meier survival estimates were computed for LC, DSS, RFS, and OS. For all analyses, statistical significance was indicated at a $p$ value of less than .05 .

The impact of prognostic variables on LC, DSS, RFS, and OS was investigated through univariate and multivariate Cox proportional hazard models. The 95\% confidence intervals (CIs) were calculated for estimates of the respective hazard ratios (HRs). The assumption of proportional hazards was assessed using (1) estimated -ln (-ln) survivor curves over different categories of variables being investigated, (2) goodness-of-fit tests based on the Schoenfel residuals, and (3) time-dependent covariates. In cases in which the proportional hazard assumption was violated, an extended Cox model was applied with the use of Heaviside functions that allow a different HR for a time-dependent variable before and after an empirically selected cutoff time point.

\section{RESULTS}

\section{Patient characteristics}

One hundred fourteen cases of OSCC were identified in our TLM database. Fifteen of the original 114 cases presented with recurrent tumors. Four cases presented as second primaries of the oral cavity and/or had history of head and neck radiation for the index primary. Thus, a total of 19 cases were excluded, leaving 95 patients consecutively treated by TLM from May 1995 to July 2010 who met entry criteria.

There were 56 men and 39 women with a median (minimum-maximum) age of 61 (range, 22-88 years) for the overall cohort, 62.5 for men (range, 35-78 years) and 59 for women (range, 22-88 years). Mean follow-up for all patients alive was 65 months, and median was 49 months (minimum-maximum $=24-204$ months).

In the entire study population, 58 patients (61\%) were alive, 56 patients (59\%) were diseasefree, and 2 patients (2\%) were alive with disease. A total of 37 patients died (39\%); 20 (21\%) from oral cancer-related causes and $17(18 \%)$ from non-oral cancer-related causes. The causes for these 17 non-oral cancer-related deaths were: cardiopulmonary $(n=6)$, pneumonia $(n=1)$, AIDS $(n=1)$, second primaries in the head and neck $(n=2)$, colon cancer $(n=1)$, traumatic brain injury $(n=1)$, burn-related injuries $(n=2)$, lung cancer $(n=$ $2)$, and hepatocellular carcinoma $(n=1)$. 
Demographic, tumor, pathologic, and treatment data are displayed in Table 1 and reflect a composition consistent with tertiary referral center practices (eg, 20\% were classified as being immunocompromised).

\section{Tumor characteristics}

Tumor site and T classification-The oral tongue was the most common site of the primary tumor $(n=59)$ followed by FOM $(n=12)$, retromolar trigone $(n=12)$, lower alveolar ridge $(n=7)$, upper alveolar ridge $(n=3)$, and buccal mucosa $(n=2)$. For the analysis, the tumor site was classified into 3 groups: (1) oral tongue, (2) FOM, and (3) "others" that comprised the remaining oral subsites $(n=25)$.

There were $47 \mathrm{~T} 1$ (49\%), $24 \mathrm{~T} 2$ (26\%), $11 \mathrm{~T} 3(11 \%)$, and $13 \mathrm{~T} 4$ (14\%) primaries. Of the $\mathrm{T} 4 \mathrm{~s}, 7$ were oral tongue primaries with involvement of deep extrinsic musculature, 5 were retromolar trigone primaries with invasion of the mandibular cortex, and 1 was upper alveolar ridge carcinoma with invasion of maxillary bone.

\section{Treatment}

Transoral laser microsurgery and neck dissections-Complete primary tumor resection through an exclusively transoral approach was achieved in 94 of the 95 patients (99\%). One patient required conversion to an open procedure for complete margin negative resection of the primary tumor. Transoral access was good in all except 3 patients, of which 1 included the afore-mentioned patient who required conversion to an open procedure. The surgical defect was reconstructed with adjacent tissue rearrangement or allowed to heal by secondary intention in 71 patients. Reconstruction was done with an acellular cadaveric dermal graft in 9 patients, split skin graft in 9 patients, and radial forearm free flap in 6 patients $(6 \%)$.

Neck dissections were performed in 48 patients (50\%), ipsilateral in 39, and bilateral in 9, 2 of whom were staged. The mean and median hospital stay was 3.4 and 2 days, respectively, with a minimum of 0 and a maximum of 16 days.

Margins-Negative histological margins were achieved in 85 of 95 patients (89.5\%). Ten patients $(10.5 \%)$ were reported to have positive surgical margins; re-resection was performed in 5 patients with 2 re-resection procedures performed in 1 patient. Of the 5 patients under-going re-resection, cancer was detected in 2; the other 3 patients had no detectable residual tumor. Re-resection was not performed in 5 patients. Of these 5 patients, 2 refused re-resection and any adjuvant therapy and are both alive and disease-free. Adjuvant therapy was planned for the remaining 3 patients ( 1 refused re-resection, 2 were unfit for the second procedure under general anesthesia) of which 1 died because of noncancer-related causes, 1 because of aggressive regional recurrence, and another immunocompromised patient because of a rapidly progressive recurrence at the primary site before initiation of adjuvant therapy. The impact of margin positivity was statistically analyzed as: (1) initial margin (ie, positive margins after the first resection; $n=10$ ) and (2) final margin (ie, positive margin after the re-resections).

Complications-There were no perioperative deaths in our series. TLM-related complications included postoperative oral bleeding in 3 patients (3\%) who needed operative intervention. After neck dissection, neck hematoma occurred in 2 patients, 1 was managed conservatively and the other operatively. One patient developed neck wound infection followed by medication-induced delirium. Chyle leak occurred in 1 patient and was managed intra-operatively with no postoperative leak. There were 4 patients with marginal mandibular nerve weakness and all recovered within a period of 6 months. Significant 
restriction of shoulder movement was recorded during the follow-up visits in 4 patients, with improvement occurring in all 4 over a period of 12 months. Another patient had a stroke 3 days after surgery.

Adjuvant therapy-Seventy-three percent of the patients ( $n=68$ of 95) did not receive any adjuvant therapy. Of the 27 patients $(27 \%)$ receiving adjuvant therapy, 17 had radiotherapy alone and 10 had concurrent chemoradio-therapy. Nine of the 17 patients who received radiotherapy alone had neck indications (ECS, multiple nodes), 5 had perineural invasion, 1 had angioinvasion, 1 had pT3N0, and 1 had margins less than $1 \mathrm{~mm}$ on the permanent pathology evaluation of the resection specimen, which also showed discontinuous and widely scattered foci of OSCC. The radiation dose administered was 66 Gy in 14 patients, 60 to $61 \mathrm{~Gy}$ in 6 patients, $70 \mathrm{~Gy}$ in 4 patients, and $72 \mathrm{~Gy}$ in 1 patient. The dosage could not be determined for 1 patient. Of the 10 patients with concurrent chemoradiotherapy, 9 had ECS and 1 had pT4N2 disease. Nine patients received cisplatin and 1 received cetuximab.

\section{Survival outcomes}

Figure 1 represents the Kaplan-Meier survival curves for LC, DSS, RFS, and OS. The 3year and 5-year survival estimates by T classification are presented in Table 2. KaplanMeier estimates for LC by T classification are illustrated in Figure 2A.

\section{Analysis of prognostic variables}

Univariate Cox regression analyses (Table 3) were performed followed by multivariate analyses for which several models were tested, with different combinations of variables. Different models were tested for multivariate analyses because of: (1) limited number of events ( $n=18$ for LC, and 20 for DSS), (2) correlation of T classification with TNM stage (chi-square $=0.000), \mathrm{N}$ classification with TNM stage (chi-square $=0.000)$, and of perineural invasion with $\mathrm{T}$ classification (chi-square $=0.002), \mathrm{N}$ classification (chi-square $=$ 0.018 ), and TNM stage (chi-square $=0.000$ ). Each multivariate model contained no more than 3 independent variables because of the limited events. No serious violation of time invariance assumption was observed. The HRs reported in Table 4 were selected based on estimation precision (95\% CIs) and significance ( $p$ values).

\section{Local control}

Univariate analysis-Comorbidity, immune compromise, and final margins were negatively prognostic for LC (Figure 2, Table 3).

Multivariate analysis-Of the 3 variables found prognostic in univariate analysis, immune compromise $(\mathrm{HR}=2.9 ; 95 \% \mathrm{CI}=1.07-7.87 ; p=.037)$ and final margins $(\mathrm{HR}=$ $6.75 ; 95 \% \mathrm{CI}=1.24-36.71 ; p=.027)$ maintained their prognostic significance as negative predictors of LC (Table 4).

\section{Disease-specific survival}

Univariate analysis-Several prognosticators had a significant HR in the univariate analyses using DSS as an end-point. These included comorbidity, immune compromise, high T classification, nodal metastasis, advanced TNM stage, final margin, $>1$ metastatic node, increasing tumor depth, tumor depth $>4 \mathrm{~mm}$, tumor grade, and perineural invasion (Figure 3, Table 3).

Multivariate analysis-Immune compromise, final margin, nodal metastasis, high T classification, and advanced TNM stage were independently prognostic for reduced DSS in 
multivariate models (Table 4). Perineural invasion was prognostic in models adjusting for immune compromise and for final margin. Comorbidity was prognostic only in a model adjusting for $\mathrm{N}$ classification. Variables of tumor grade, tumor depth, and $>1$ metastatic node could not be tested in multivariate analysis because of limited data points.

\section{Recurrence-free survival}

The same variables prognostic for DSS, with the exception of high T classification, final margin, and tumor depth $>4 \mathrm{~mm}$, were significant for predicting reduced RFS in univariate analysis (Table 3). The results for multivariate analysis are presented in Table 4.

\section{Overall survival}

Presentation of OSCC as a second primary, comorbidity, immune compromise, high T classification, nodal metastasis, advanced TNM stage, final margin, $>1$ meta-static node, increasing tumor depth, tumor grade, and perineural invasion were all associated with a reduced OS in univariate analysis. Factors that retained their significance in multivariate analysis are reported in Table 4.

\section{Other prognostic variables}

Status of surgical margins after initial resection was not prognostic for any of the disease outcomes; however, the final margin status after re-resection was prognostic for all. The frequency of local recurrence in the initial margin positive group was $20 \%(n=2$ of 10$)$ versus $18.8 \%(n=16$ of 85$)$ in the initial margin negative group, whereas it was $40 \%$ in the final margin positive group ( $n=2$ of 5$)$ versus $18 \%(n=16$ of 90$)$ in the final margin negative group. Age, sex, tumor site, smoking status, ECS, soft tissue metastasis, lymphovascular invasion, advancing front of the tumor, inflammatory response of the tumor, and absence of adjuvant therapy were not prognostic.

\section{Patterns of recurrence}

Recurrences occurred in a total of 36 patients (37\%), 14 of whom recurred at more than 1 site. Recurrence sites was distributed as 18 local, 18 regional, and 16 distant in the 36 patients. Five patients had highly aggressive disease that recurred within a month of surgery before receiving adjuvant therapy.

\section{Time to recurrence}

Seventy-five percent of the recurrences $(n=27$ of 36) occurred within the first year after primary surgery, 4 in the second year, 3 in the third year, and 1 in the fourth year. One patient with a 90 pack-year smoking history developed a solitary lung squamous cell carcinoma lesion at 93 months from TLM resection, which may well have been a second lung primary, but because of the lack of evidence to differentiate, was counted as distant metastasis for analysis.

The local recurrences occurred from 1 to 38 months (median, 6.4 months). Regional recurrences were detected between 2 and 26 months (median, 9 months), whereas all distant metastases were detected between 5 and 28.7 months, excluding the case at 93 months (median, 10.3 months).

\section{Management of recurrences}

Surgical salvage was performed in 14 of 18 patients with local recurrence (5 TLM, 9 open). It failed to control the primary in 5, and was successful in 9 cases (4 TLM, 5 open) for a final absolute LC rate of $91 \%$. Of these salvaged patients, there were no local re-recurrences, 4 are alive and disease-free, 3 died because of distant metastasis, and 2 because of non- 
cancer-related causes. Final regional control was 91.6\%, achieved with neck dissection in 10 of the 18 patients with regional recurrences. At the last follow-up, 4 patients were alive and disease-free, 1 died of local recurrence, 2 died of distant metastasis, and 3 because of noncancer-related causes. Two of the 16 patients with pulmonary distant metastasis underwent wedge resection and were disease-free at the last follow-up.

\section{Functional outcomes}

Airway-Eighty patients did not have a tracheostomy at any stage of their treatment or follow-up. Six needed a temporary tracheostomy within 30 days of surgery, and all were successfully decannulated. Eight needed a tracheotomy after 30 days of surgery either for recurrent disease or other causes. One patient had a preexisting tracheostomy performed several years ago when he underwent surgery for supraglottic cancer.

Swallowing -No feeding tube was required for 53 patients (57\%). Fourteen had nasogastric tubes whereas 28 needed gastrostomy tubes at some stage during their treatment or follow-up course. Only 6 of the patients had long-term gastrostomy, all had recurrences.

The FOSS scores at last available follow-up are available for 81 of 95 patients (85\%) in our series. The data could not be collected for 14 patients. The median FOSS score (0-5) for these 81 patients was 0 . The distribution of FOSS score was FOSS 0 in 53, FOSS 1 in 15, FOSS 2 in 3, and FOSS 4 in 5 patients. Five patients had a FOSS score of 5, all of which had disease recurrence.

\section{DISCUSSION}

Within the constraints of this aggressive tumor phenotype, our study demonstrates that transoral laser microsurgery is an effective surgical treatment approach for OSCC. Applied to the full $\mathrm{T} 1$ to $\mathrm{T} 4$ spectrum of OSCC primaries (75\% T1-T2s and 25\% T3-T4s), TLM resection achieved a 5-year LC of 78\% (Kaplan-Meier) for the overall cohort, 78\% for T3s, and $69 \%$ for the T4s, respectively.

The DSS at 5-years for our entire cohort was $76 \%$ and OS at 5-years was 62\%. These estimates are expected, given the high proportion of the study population having advanced TNM III or IV disease (44\%) and corresponds to our finding that advanced TNM stage is an independent prognosticator. To provide context, in non-TLM surgical series with similar ${ }^{14,15}$ advanced disease proportions, the estimates for 5-year OS estimates range from $48 \%$ to $71 \%, 14,15$ whereas disease-related survival outcomes range between $42 \%$ and $76 \%$ (Table 5). ${ }^{14,15}$ One of these non-TLM studies for OSCC reports DSS estimates of $76 \%,{ }^{13}$ similar to the current study; however, the proportion of advanced-stage tumors was roughly one-half $(23 \%)^{13}$ of that in our study. Non-TLM studies with higher proportions of advanced-stage cases demonstrate 5 -year OS of $49 \%{ }^{11}$ to $56 \%,{ }^{12}$ and using disease-specific estimates that count only death from oral cancer, such series report estimates at $49 \% 12$ and $74 \%{ }^{11}$ (Table 5).

Compared to a previously reported all-inclusive, T1 to T4, TLM series by Eckel et al ${ }^{10}$ ( $n=$ 64), the current study had comparable DSS estimates for stages I to III, and slightly better outcomes for stage IV lesions ( $31 \%$ vs $21 \%,{ }^{10}$ respectively). Unlike our study, all neck dissections in the Eckel et al ${ }^{10}$ series were staged.

In the context of other recent cohort studies, it is therefore reasonable to conclude that the TLM treatment approach taken in this cohort gave results that support it's continued use for primary tumor management. 


\section{Local control}

A total of 18 patients $(19 \%)$ had recurrence at the primary site, with surgical salvage achieved in 9 of the 18 patients, for an absolute final LC of $91 \%$. Thus, transoral resection cannot only be applied as initial definitive treatment for the primary tumor, but also preserves re-treatment local salvage options in the form of further surgery (open or transoral) and/or radiotherapy. In contrast, non-TLM surgery, despite also demonstrating reasonable LC rates (87\%), ${ }^{11}$ may involve greater neck or facial tissue disruption and render effective revision surgery more difficult. Salvage surgery cure rates in non-TLM surgical series have been reported in the range of $15 \%$ to $67 \% .^{22}$

On comparing LC rates with a large non-TLM surgery series, ${ }^{15}$ albeit with lower proportions of T3 to T4 tumors than our study ( $16 \%{ }^{15}$ vs $\left.25 \%\right)$, we find a similar LC rate ( $78 \% 15$ vs $78.4 \%$ in our series). Our outcomes also compare favorably when stratified by $\mathrm{T}$ classification: $84 \%$ vs $89 \%^{15}$ for T1; $74 \%$ vs $69 \%^{15}$ for T2; $78 \%$ vs $62 \%{ }^{15}$ for T3; and $69 \%$ vs $71 \%{ }^{15}$ for T4. The use of adjuvant therapy was similar; $27 \%$ in our study vs $25 \%{ }^{15}$

\section{Regional control and flap considerations}

A total of $18(19 \%)$ regional recurrences were observed. Ten of the 18 regional recurrences were salvaged with further neck dissection for a final regional nodal control of $91.6 \%$. Although TLM clearly does not address regional control, it leaves the neck tissues undisturbed during the resection of the primary. Furthermore, despite some evidence supporting incontinuity neck dissection for oral cavity malignancy, ${ }^{23}$ we have not found high recurrence rates attributable to discontinuity of the primary resection and the neck dissection. This approach keeps the neck dissection confined to its own field and reduced the need for free flap reconstruction, which was performed in only $6 \%$ of our patients.

Our reconstructive decisions were defect-based e.g. (location relative to mobile structures, bone exposure), rather than $\mathrm{T}$ classification driven. The free flap reconstruction rate may reach $76 \%{ }^{11}$ in non-TLM series despite small differences in the proportion of advanced stage tumors $\left(56 \%{ }^{11}\right.$ vs $\left.44 \%\right)$ and/or T3 to 4 primaries (39\% ${ }^{11}$ vs $\left.25 \%\right)$ from the current study. In another non-TLM study with a free flap reconstruction rate of $77 \%,{ }^{13}$ the proportion of advanced stage tumors was $23 \%{ }^{13}$ (Table 5). Although many unreported variables certainly influence reconstructive choices, rendering comparison between series difficult, it is intuitively and clinically reasonable that a discontinuity approach, frequently leaving the mylohyoid muscle intact, obviates the need for regional or free flap reconstruction. Inset of the free flaps we did use in our patients did not entail additional incisions or major normal tissue disruption: inset, usually with multidimensional folding 24 was performed transorally, and a short tunnel was bluntly created to the neck for exit of the neurovascular pedicle using the incision and exposure created by the concomitant neck dissection. A typical end result is seen in Figure 4.

\section{Prognostic factors}

For the disease-related outcomes of DSS and RFS, we found a set of prognosticators consistent with other published studies on oral cancer, such as high T classification, advanced TNM stage, presence of nodal metastasis, and perineural invasion. ${ }^{11-15}$ Host factors, such as moderate to severe comorbidity and immune compromise, were also independent prognosticators. Adjuvant therapy was nonprognostic for all primary and secondary endpoints. Lower frequency of recurrences was noted in patients who did not receive adjuvant therapy compared with patients with adjuvant therapy $(31 \%$ vs $55 \% ; p=$ 0.025 ), although it is likely that there was selection bias for adjuvant therapy in advanced cases. 
Among all patient, tumor, and treatment-related variables, on multivariate analysis, immune compromise was significantly associated with reduced LC and each survival endpoints chosen. The absolute frequency of local recurrences in patients with immune compromise ( $n$ $=7$ of $19 ; 37 \%)$ differed significantly $(p=0.04)$ from patients without immune compromise $(n=11$ of $76 ; 14 \%)$. This underscores the critical role of host immunity for LC and survival in oral cavity cancer and highlights the urgent need for research that characterizes and quantifies host immunity to this disease. New treatments to enhance host immunity are clearly needed and in OSCC may hold as much promise as tumor molecular targeting. Adjuvant therapy that is immunosuppressive should also therefore be used judiciously.

\section{Status of surgical margins}

After the initial primary resection, negative margins were achieved in 85 of 95 patients $(89.5 \%)$. No residual tumor was observed in 3 of the 5 re-resections, and the remaining 2 were re-resected to negative margins. Thus, the final margin positive rate, albeit only unverified by re-resection, was $5 \%(n=5$ of 95$)$. Verified final negative margins was achieved in $95 \%$ of patients. Given the absolute rate of local recurrence observed (19\%; $n=$ 18 of 95), the observation of at least $95 \%$ verified margin negativity forms the premise to investigate factors other than margin status that may better predict local recurrence.

In the TLM setting, sampling, labeling, and inking of margins on the main tumor and the frozen specimens assumes critical importance and needs to be meticulously observed by the surgeon. Higher margin positivity has been documented in TLM studies on oral cancer in the absence of frozen sections. ${ }^{10}$ Eckel et al ${ }^{10}$ reported a margin positivity rate of $37 \%$, although residual tumor could be found in only $15 \%$ of the re-resections. This was attributed to nonavailability of frozen sections during the surgery. Compared to en bloc approaches, TLM may have a unique beneficial impact on mitigating the widely recognized negative impact of positive margins in oral cavity cancer surgery. Lower rates of margin positivity $(10.5 \%)$ than non-TLM techniques $(17 \%)^{11}$ after initial resection in this series, with final positivity in only $5 \%$, are uniquely facilitated by TLM because multiple points of assessment of tumor depth and spread are made with transtumoral cuts. This approach increases precision, and the probability of achieving a negative margin, particularly the deep margin. Unique to the TLM technique, transtumoral laser incisions allow the surgeon, in a dry field, to identify the peripheral tumor margins. The powerful magnification, resolution, and illumination of an operating microscope facilitate clear distinctions between healthy versus tumor tissue and sometimes demonstrate unexpected tumor extensions (eg, those occurring along/within the lingual nerve) without loss of excessive normal mucosa. This is in contrast to conventional en bloc surgery in which only the pathologist ever sees the deep surface of the tumor, usually after surgery is completed and considerable risk persists of unanticipated positive or close margins. These latter events also upscale patients to "high risk," who often then receive normal tissue-toxic levels of adjuvant therapy to the primary site.

\section{Functional outcomes and complications}

Functional outcomes for oral cavity surgery require measures of oral competence, mastication, articulation, and swallowing. Although each of these was not analyzed separately, we captured functional results expressed as swallowing scores to give a global assessment of nutritional competence. The FOSS scores in this study indicated that the majority of patients (FOSS $0-2 ; n=71$ of $95 ; 75 \%$ ) achieved satisfactory oral nutritional intake.

Airway compromise may occur after oral cavity surgery, especially if the suspensory muscles of the larynx (eg. the geniohyoids) are detached. Therefore, tracheotomy and 
decannulation rates are other reasonable parameters for assessment of functional outcomes after TLM. Our series had a $6 \%$ temporary tracheotomy rate.

Regarding complications, oral bleeding was the only adverse event directly attributable to TLM. With 3 cases of bleeding (3\%), all controlled successfully, we consider this acceptable, and in the same range as reported for post-tonsillectomy hemorrhage.

\section{Limitations}

This is a single, tertiary institution study that has its own inherent selection biases. Furthermore, because this was not a comparative study, we cannot make definitive statements regarding advantages or disadvantages of TLM over non-TLM surgical approaches. Also, the validity of the prognostic factors may be limited or changed in the future because of greater sample sizes and the retrospective analyses. However, to our knowledge, this is the largest identifiable TLM series to date encompassing all stages of OSCC. Finally, there may be additional events in the form of recurrences or deaths if the minimum follow-up period of 2 years was extended.

\section{CONCLUSIONS}

We document a series of OSCC treated with TLM and simultaneous neck dissection, when indicated. A relatively high proportion of stage III/IV cases (44\%) was observed; one fourth of the cohort had T3 to T4 primaries. This demonstrates the feasibility of TLM application across all stages with an absolute LC rate of over $90 \%$, effective retreatment options, acceptable survival outcomes, and optimum function. Moreover, the free tissue transfer was low. Using this minimally invasive TLM approach, we observed a maximum of 5\% positive margins, an oncologically important result. However, there was a local recurrence rate of $19 \%$, and a strong correlation was seen with the presence or history of immune compromise. This observations warrants further exploration of potential tumor or host factors that may impact LC rates in TLM-treated oral cancer cohorts. With the above results, we continue to recommend and practice the transoral laser microsurgery approach in management of suitable cases of primary oral cavity carcinomas.

\section{Acknowledgments}

The authors thank Debbie Turner, Department of Otolaryngology, for her help with data management.

\section{REFERENCES}

1. Shah JP, Gil Z. Current concepts in management of oral cancer-surgery. Oral Oncol. 2009; 45:394401. [PubMed: 18674952]

2. Mihashi S, Jako GJ, Incze J, Strong MS, Vaughan CW. Laser surgery in otolaryngology: interaction of CO2 laser and soft tissue. Ann N Y Acad Sci. 1976; 267:263-294. [PubMed: 1064357]

3. Spiro RH, Strong EW. Discontinuous partial glossectomy and radical neck dissection in selected patients with epidermoid carcinoma of the mobile tongue. Am J Surg. 1973; 126:544-546. [PubMed: 4743841]

4. Strong MS, Jako GJ. Laser surgery in the larynx. Early clinical experience with continuous CO 2 laser. Ann Otol Rhinol Laryngol. 1972; 81:791-798. [PubMed: 4636137]

5. Strong MS, Vaughan CW, Healy GB, Shapshay SM, Jako GJ. Transoral management of localized carcinoma of the oral cavity using the CO2 laser. Laryngoscope. 1979; 89(6 Pt 1):897-905. [PubMed: 449535]

6. Panje WR, Scher N, Karnell M. Transoral carbon dioxide laser ablation for cancer, tumors, and other diseases. Arch Otolaryngol Head Neck Surg. 1989; 115:681-688. [PubMed: 2719826] 
7. Steiner W. Results of curative laser microsurgery of laryngeal carcinomas. Am J Otolaryngol. 1993; 14:116-121. [PubMed: 8484476]

8. Haughey BH, Hinni ML, Salassa JR, et al. Transoral laser microsurgery as primary treatment for advanced-stage oropharyngeal cancer: a United States multicenter study. Head Neck. 2011; 33:1683-1694. [PubMed: 21284056]

9. Jerjes W, Upile T, Hamdoon Z, Mosse CA, Akram S, Hopper C. Prospective evaluation of outcome after transoral CO2 laser resection of T1/T2 oral squamous cell carcinoma. Oral Surg Oral Med Oral Pathol Oral Radiol Endod. 2011; 112:180-187. [PubMed: 21232996]

10. Eckel HE, Volling P, Pototschnig C, Zorowka P, Thumfart W. Transoral laser resection with staged discontinuous neck dissection for oral cavity and oropharynx squamous cell carcinoma. Laryngoscope. 1995; 105:53-60. [PubMed: 7837914]

11. Rogers SN, Brown JS, Woolgar JA, et al. Survival following primary surgery for oral cancer. Oral Oncol. 2009; 45:201-211. [PubMed: 18674959]

12. Aksu G, Karadeniz A, Saynak M, Fayda M, Kadehci Z, Kocaelli H. Treatment results and prognostic factors in oral tongue cancer: analysis of 80 patients. Int J Oral Maxillofac Surg. 2006; 35:506-513. [PubMed: 16503396]

13. Koo BS, Lim YC, Lee JS, Choi EC. Recurrence and salvage treatment of squamous cell carcinoma of the oral cavity. Oral Oncol. 2006; 42:789-794. [PubMed: 16455287]

14. Sklenicka S, Gardiner S, Dierks EJ, Potter BE, Bell RB. Survival analysis and risk factors for recurrence in oral squamous cell carcinoma: does surgical salvage affect outcome? J Oral Maxillofac Surg. 2010; 68:1270-1275. [PubMed: 20347201]

15. Goldstein DP, Bachar GY, Lea J, et al. Outcomes of squamous cell cancer of the oral tongue managed at the Princess Margaret Hospital. Head Neck. 2012 [Epub ahead of print].

16. Piccirillo JF, Tierney RM, Costas I, Grove L, Spitznagel EL Jr. Prognostic importance of comorbidity in a hospital-based cancer registry. JAMA. 2004; 291:2441-2447. [PubMed: 15161894]

17. Hyland A, Li Q, Bauer JE, Giovino GA, Steger C, Cummings KM. Predictors of cessation in a cohort of current and former smokers followed over 13 years. Nicotine Tob Res. 2004; 6(suppl 3):S363-S369. [PubMed: 15799599]

18. D'Souza G, Kreimer AR, Viscidi R, et al. Case-control study of human papillomavirus and oropharyngeal cancer. N Engl J Med. 2007; 356:1944-1956. [PubMed: 17494927]

19. Salassa JR. A functional outcome swallowing scale for staging oropharyngeal dysphagia. Dig Dis. 2000; 17:230-234. [PubMed: 10754363]

20. Fakih AR, Rao RS, Borges AM, Patel AR. Elective versus therapeutic neck dissection in early carcinoma of the oral tongue. Am J Surg. 1989; 158:309-313. [PubMed: 2802032]

21. Steiner, W.; Ambrosch, P.; Knappe, MV. Endoscopic laser surgery of the upper aerodigestive tract: with special emphasis on cancer surgery. New York, NY: Thieme Medical Publishers; 2001.

22. Schwartz GJ, Mehta RH, Wenig BL, Shaligram C, Portugal LG. Salvage treatment for recurrent squamous cell carcinoma of the oral cavity. Head Neck. 2000; 22:34-41. [PubMed: 10585603]

23. Leemans CR, Tiwari R, Nauta JJP, Snow GB. Discontinuous vs in-continuity neck dissection in carcinoma of the oral cavity. Arch Otolaryngol Head Neck Surg. 1991; 117:1003-1006. [PubMed: 1910714]

24. Haughey BH, Taylor SM, Fuller D. Fasciocutaneous flap reconstruction of the tongue and floor of mouth: outcomes and techniques. Arch Otolaryngol Head Neck Surg. 2002; 128:1388-1395. [PubMed: 12479726] 


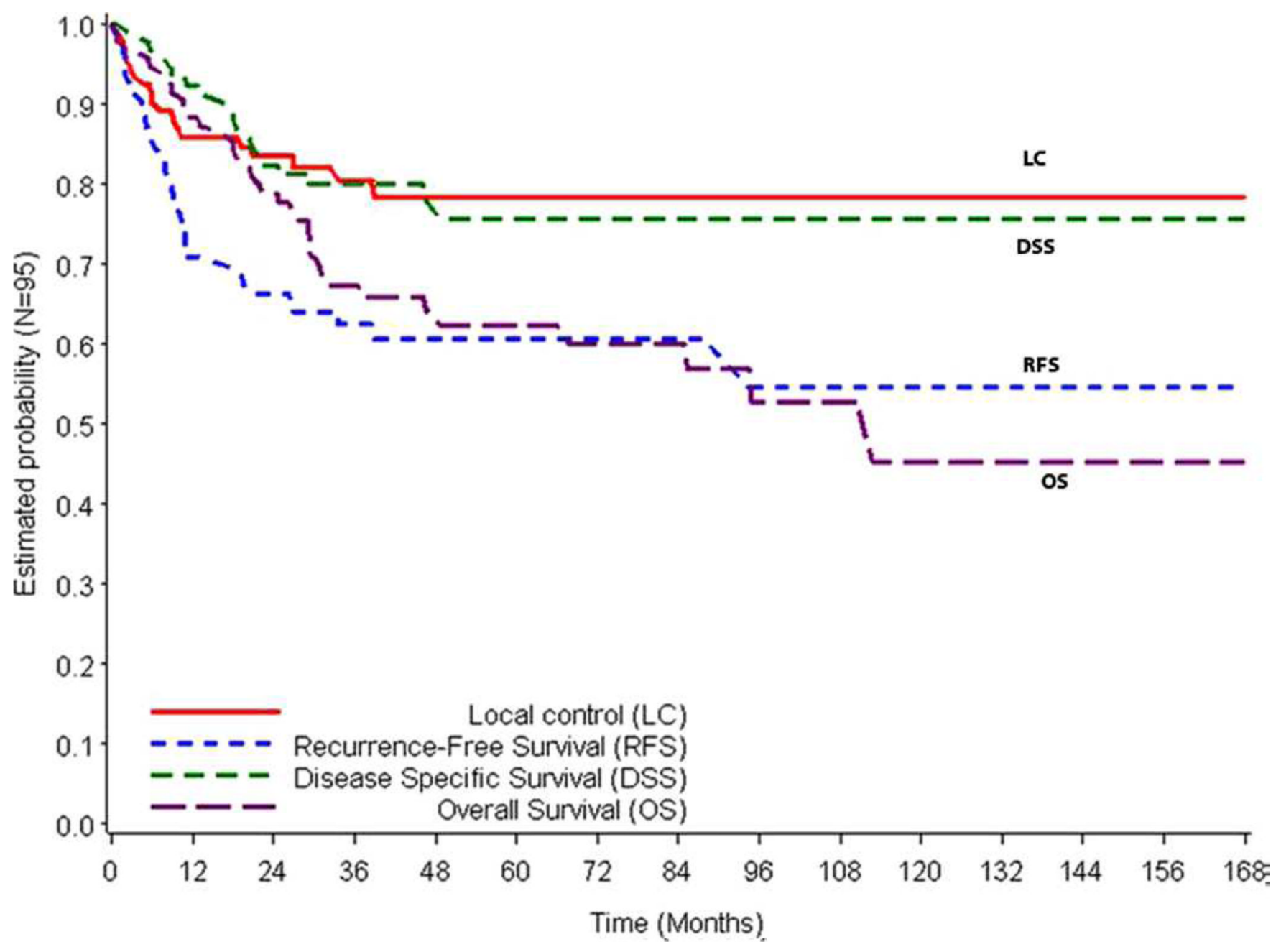

FIGURE 1.

Kaplan-Meier curves for local control (LC), disease-specific survival (DSS), recurrence-free survival (RFS), and overall survival (OS). [Color figure can be viewed in the online issue, which is available at wileyonlinelibrary.com.] 

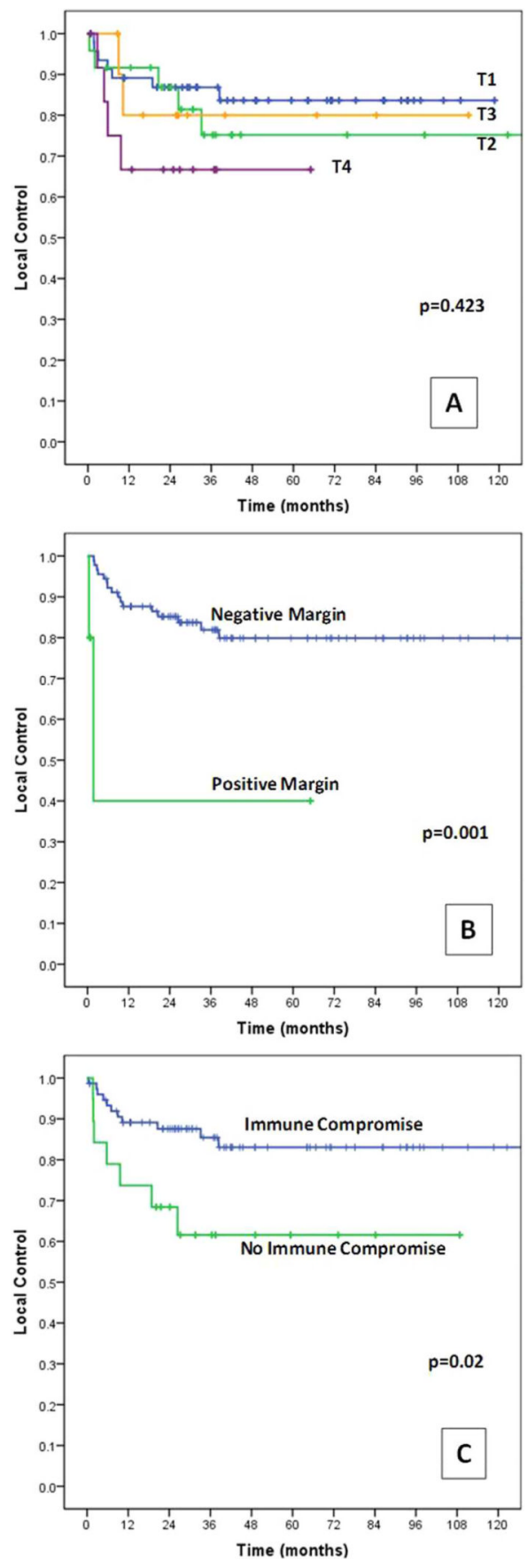

FIGURE 2.

Kaplan-Meier curves for local control by (A) T classification, (B) positive versus negative final margins, and (C) presence versus absence of immune compromise. [Color figure can be viewed in the online issue, which is available at wileyonlinelibrary.com.] 

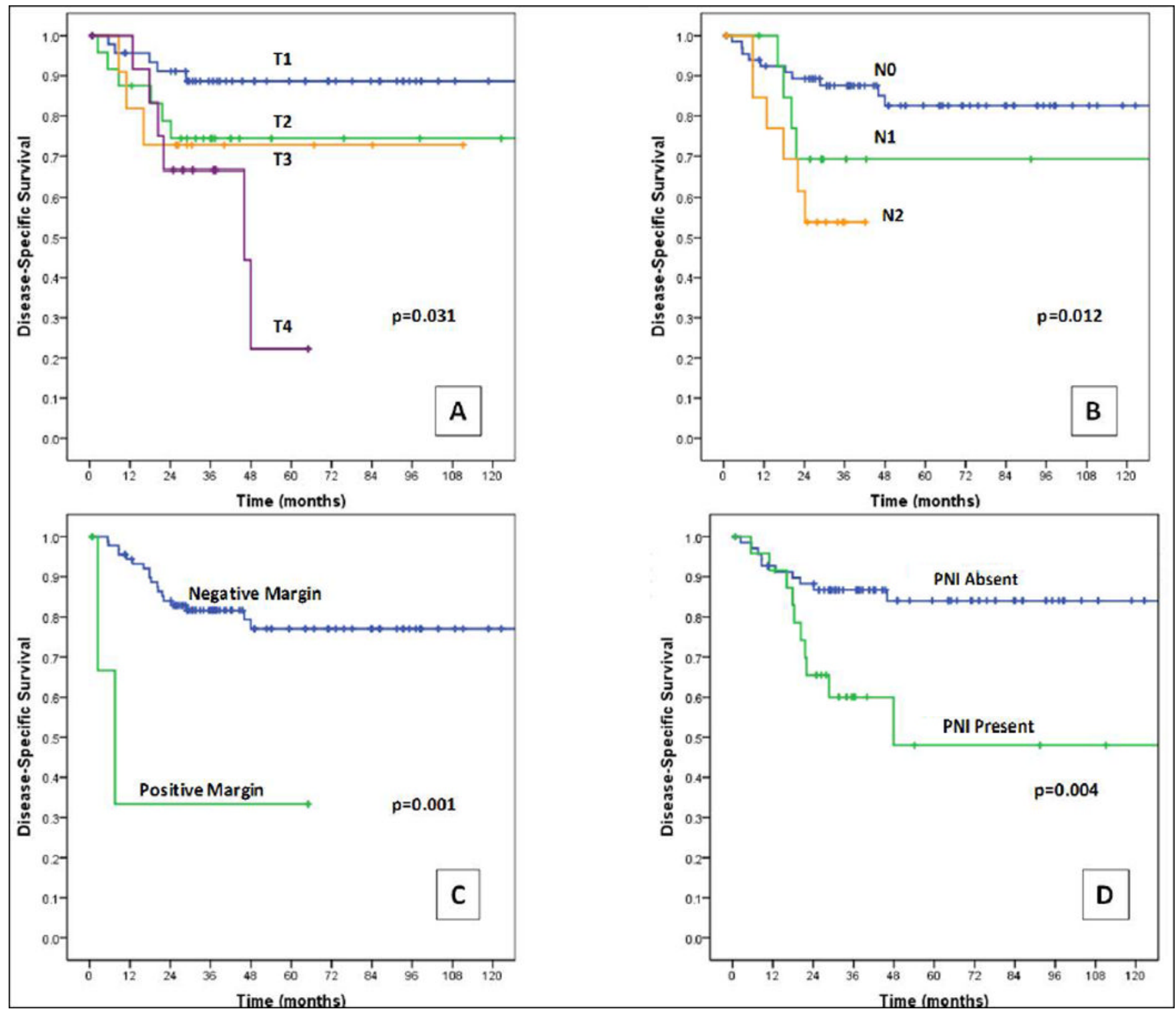

FIGURE 3.

Kaplan-Meier curves for disease-specific survival by (A) T classification, (B) $\mathrm{N}$ classification, (C) positive versus negative final margins, and (D) presence versus absence of perineural invasion (PNI). [Color figure can be viewed in the online issue, which is available at wileyonlinelibrary.com.] 

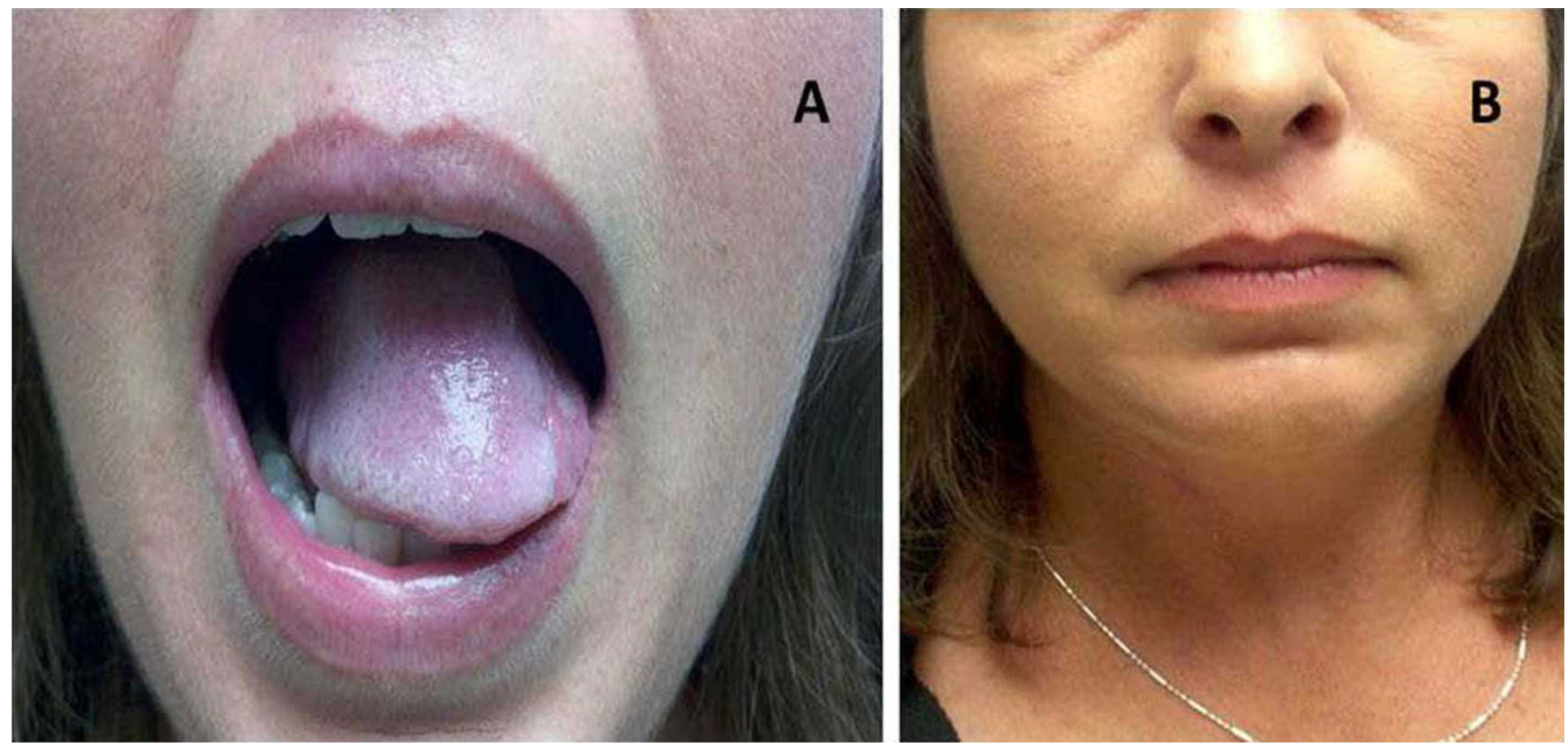

FIGURE 4.

(A) Free flap reconstruction in a 40-year-old woman after transoral laser microsurgery (TLM) resection of T3 oral tongue carcinoma, bilateral neck dissections, and transoral flap inset: approximately $85 \%$ of visible neo-tongue tissue is the flap, with a thin strip of native tongue on left, (B) cosmetic result of lips, lower face, and neck. [Color figure can be viewed in the online issue, which is available at wileyonlinelibrary.com.] 


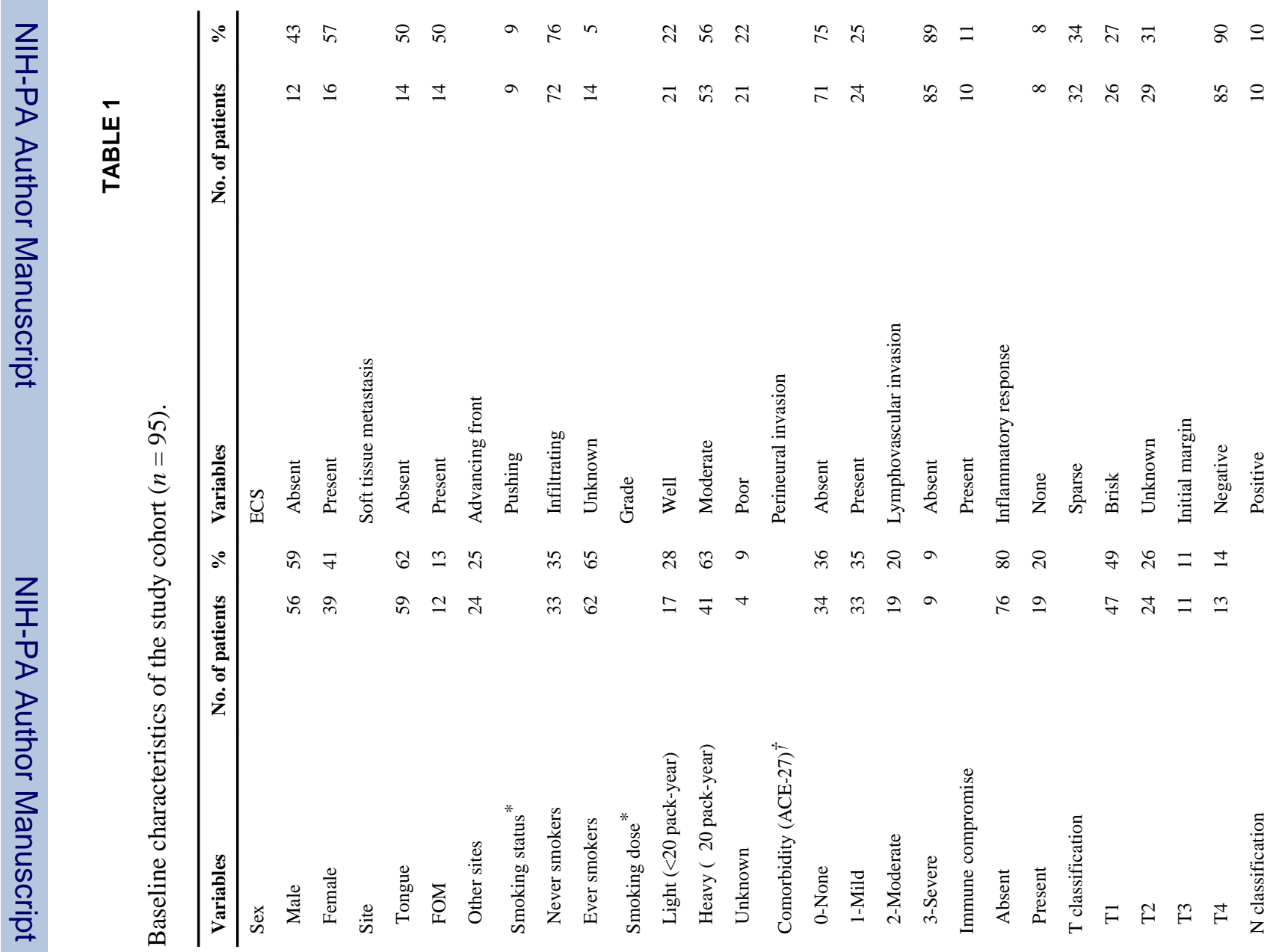
Head Neck. Author manuscript; available in PMC 2014 March 12. 


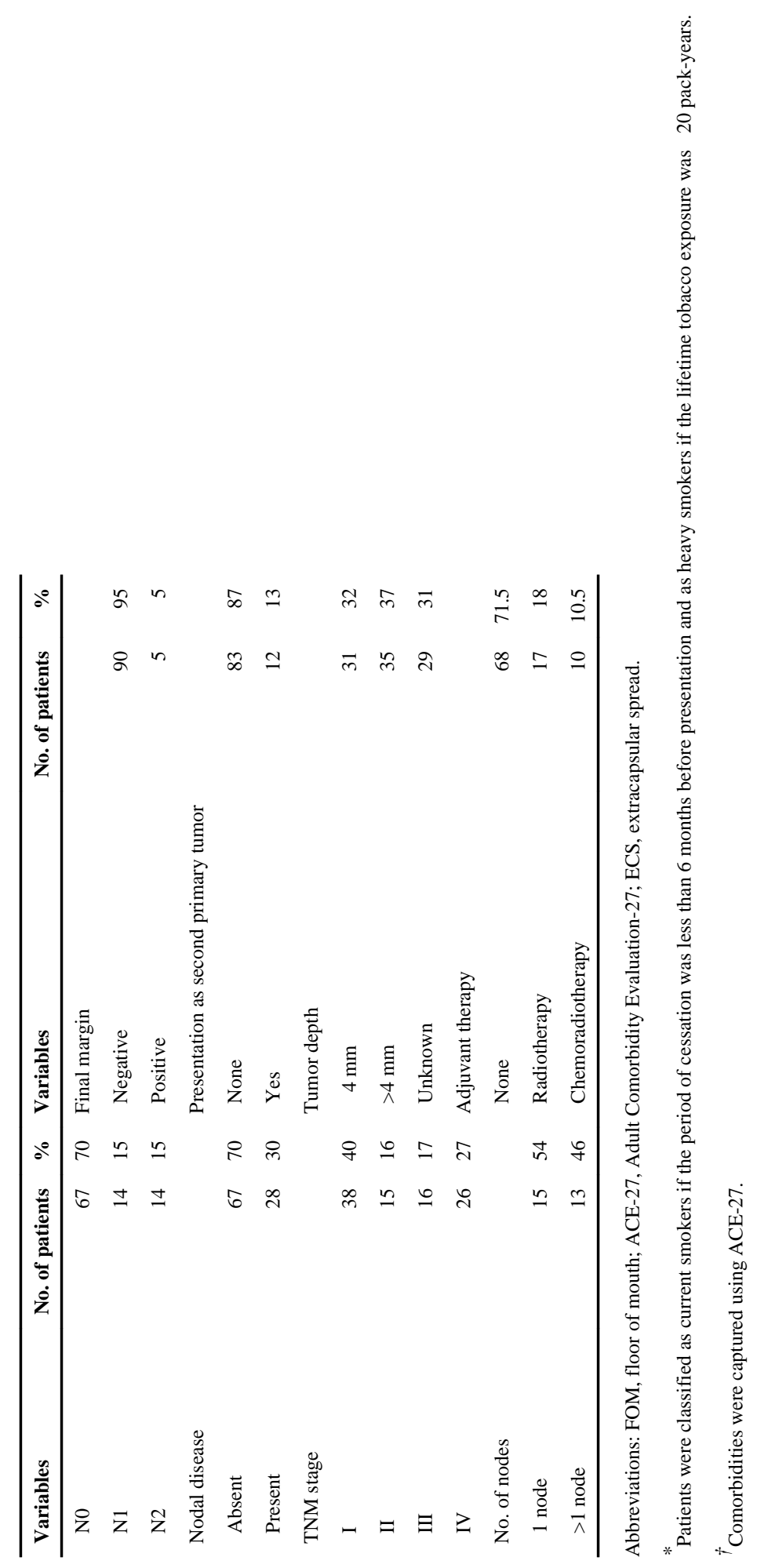




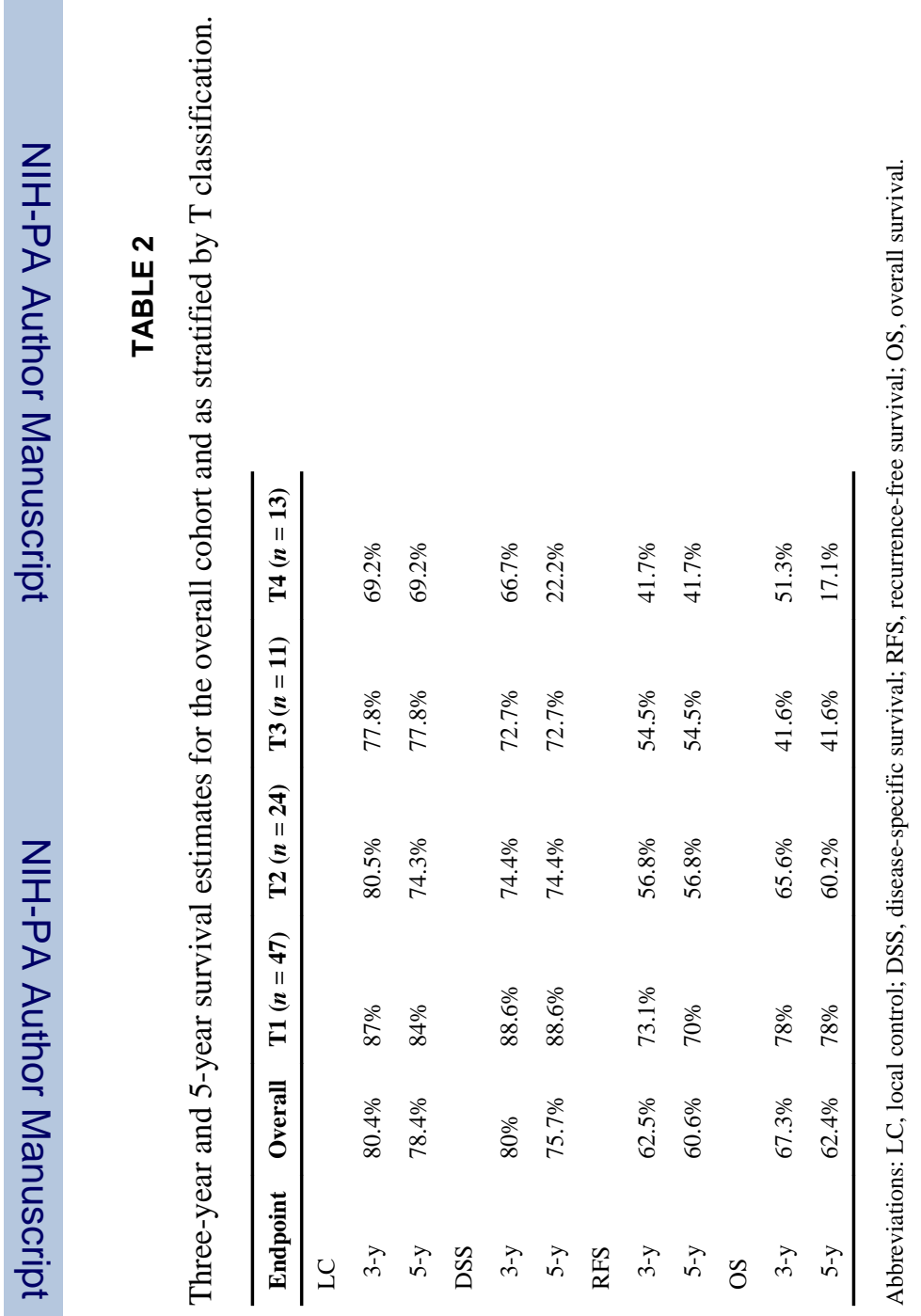




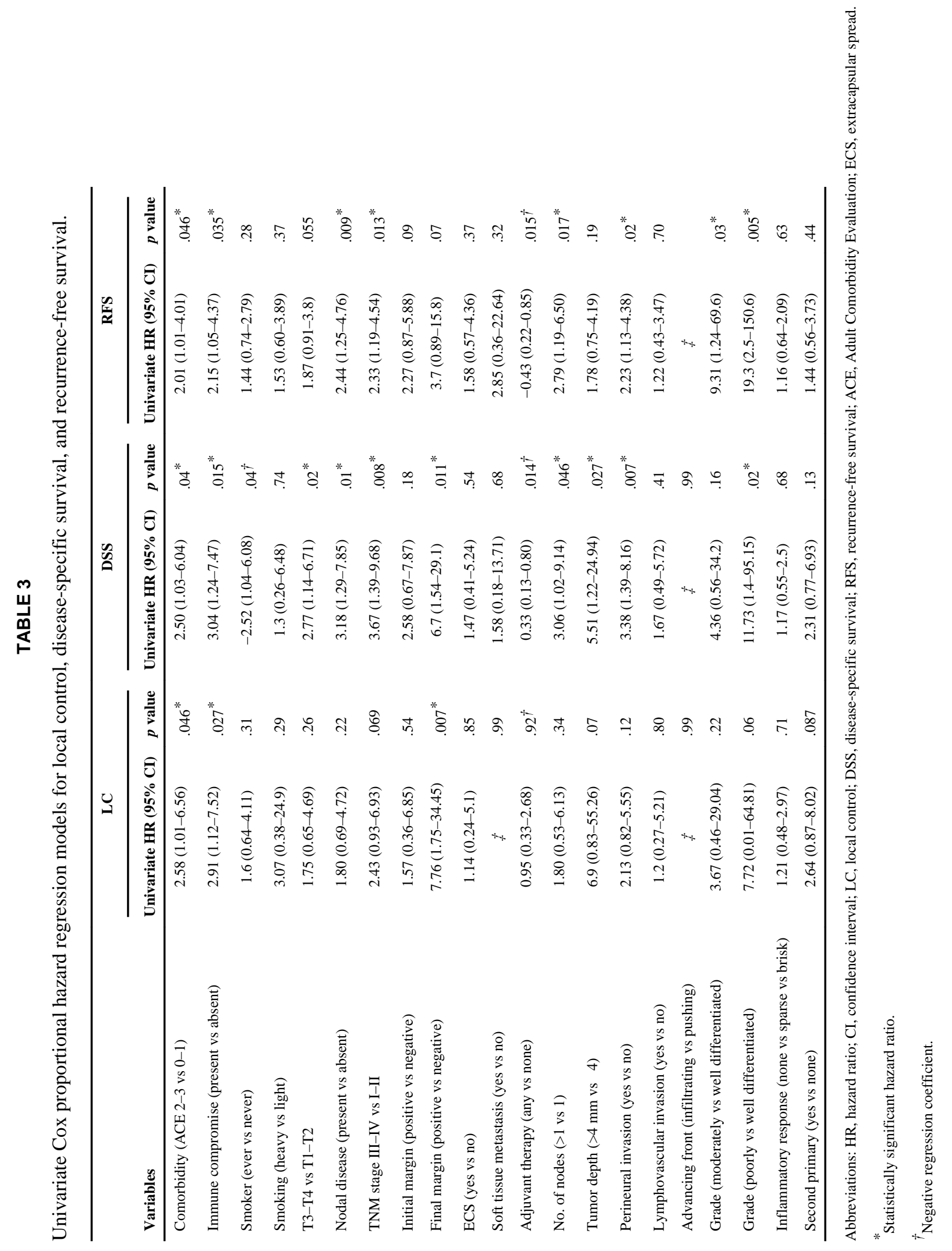

Head Neck. Author manuscript; available in PMC 2014 March 12. 
TABLE 4

Cox regression multivariate analysis for local control, disease-specific survival, recurrence-free survival, and overall survival.

\begin{tabular}{|c|c|c|c|c|}
\hline & LC HR $(95 \%$ CI $)$ & DSS HR $(95 \%$ CI $)$ & RFS HR $(95 \%$ CI $)$ & OS HR $(95 \% \mathrm{CI})$ \\
\hline \multirow[t]{2}{*}{ Immune compromise (present vs absent) } & $2.90(1.07-7.87)$ & $3.13(1.24-7.88)$ & $2.26(1.10-4.63)$ & $2.27(1.09-4.68)$ \\
\hline & $p=.037$ & $p=.016$ & $p=.026$ & $p=.027$ \\
\hline \multirow[t]{2}{*}{ Comorbidity (ACE $2-3$ vs $0-1$ ) } & NS & $2.6(1.05-6.47)$ & NS & $2.16(1.04-4.68)$ \\
\hline & & $p=.039$ & & $p=.04$ \\
\hline \multirow[t]{2}{*}{$\mathrm{T} 3-\mathrm{T} 4$ vs $\mathrm{T} 1-\mathrm{T} 2$} & NS & $3.01(1.21-7.48)$ & NS & $2.93(1.48-5.78)$ \\
\hline & & $p=.017$ & & $p=.002$ \\
\hline \multirow[t]{2}{*}{ Nodal disease (present vs absent) } & NS & $2.54(1.01-6.40)$ & $2.39(1.19-4.79)$ & $3.54(1.74-7.21)$ \\
\hline & & $p=.048$ & $p=.014$ & $p=.000$ \\
\hline \multirow[t]{2}{*}{ TNM stage III-IV vs I-II } & NS & $2.91(1.08-7.77)$ & $2.04(1.03-4.06)$ & $4.11(1.93-8.75)$ \\
\hline & & $p=.033$ & $p=.041$ & $p=.000$ \\
\hline \multirow[t]{2}{*}{ Final margin (positive vs negative) } & $6.75(1.24-36.7)$ & $5.52(1.2-25.31)$ & $5.54(1.27-24.2)$ & $5.08(1.58-16.3)$ \\
\hline & $p=.027$ & $p=.028$ & $p=.023$ & $p=.006$ \\
\hline \multirow[t]{2}{*}{ Perineural invasion (present vs absent) } & NS & $4.4(1.72-11.29)$ & $2.41(1.21-4.78$ & $2.75(1.44-5.51)$ \\
\hline & & $p=.002$ & $p=.012$ & $p=.004$ \\
\hline \multirow[t]{2}{*}{ Second primary (present vs absent) } & NS & NS & NS & $3.08(1.59-5.98)$ \\
\hline & & & & $p=.001$ \\
\hline
\end{tabular}

Abbreviations: LC, local control; DSS, disease-specific survival; RFS, recurrence-free survival; OS, overall survival; ACE, Adult Comorbidity Evaluation; NS, not significant. 


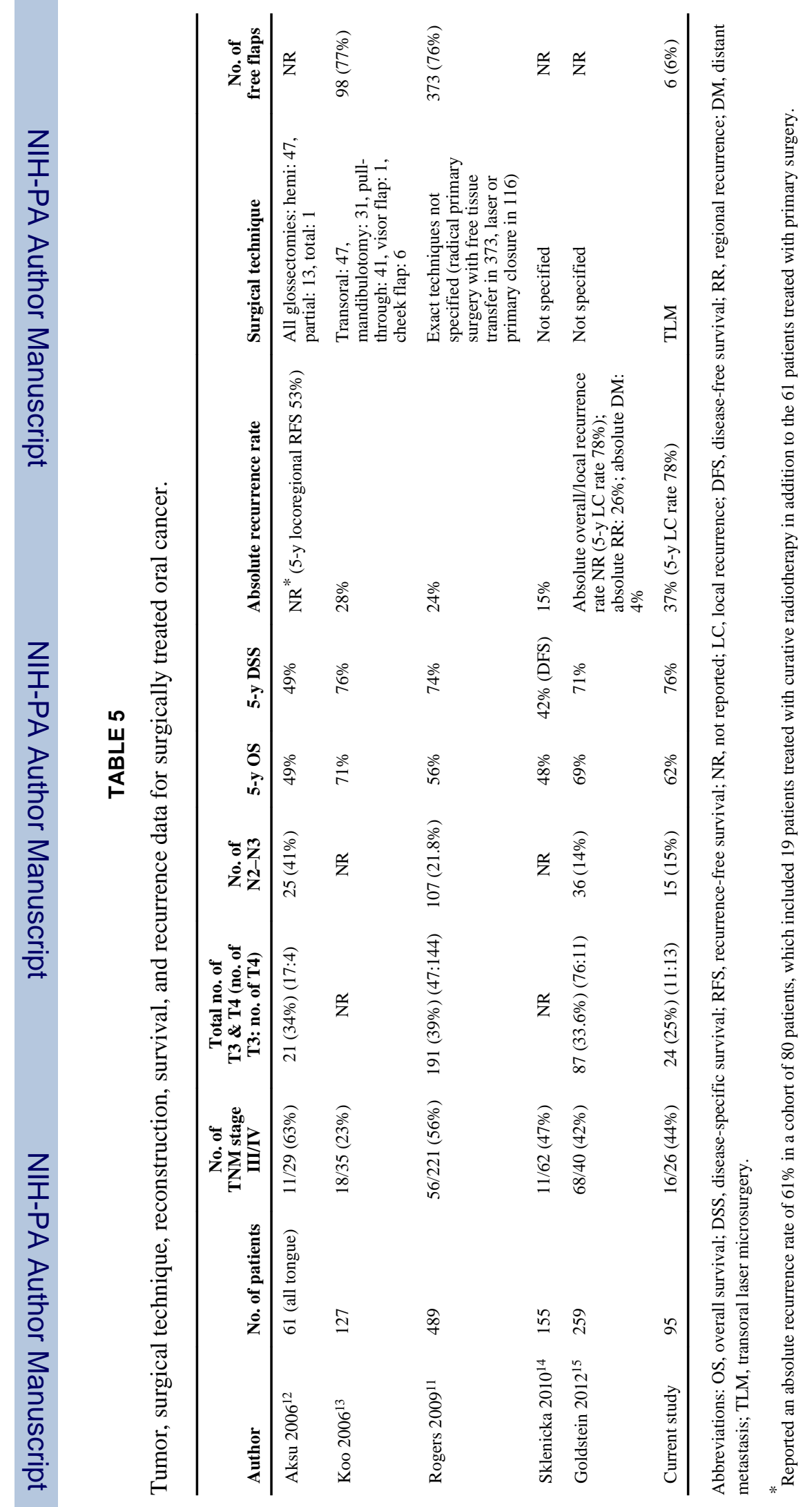

Head Neck. Author manuscript; available in PMC 2014 March 12. 